\title{
A Sharp Condition for Exact Support Recovery of Sparse Signals With Orthogonal Matching Pursuit
}

\author{
Jinming Wen, Zhengchun Zhou, Jian Wang, Xiaohu Tang and Qun Mo
}

\begin{abstract}
Support recovery of sparse signals from noisy measurements with orthogonal matching pursuit (OMP) has been extensively studied in the literature. In this paper, we show that for any $K$-sparse signal $\boldsymbol{x}$, if the sensing matrix $\boldsymbol{A}$ satisfies the restricted isometry property (RIP) of order $K+1$ with restricted isometry constant (RIC) $\delta_{K+1}<1 / \sqrt{K+1}$, then under some constraint on the minimum magnitude of the nonzero elements of $x$, the OMP algorithm exactly recovers the support of $x$ from the measurements $y=A x+v$ in $K$ iterations, where $v$ is the noise vector. This condition is sharp in terms of $\delta_{K+1}$ since for any given positive integer $K \geq 2$ and any $1 / \sqrt{K+1} \leq t<1$, there always exist a $K$-sparse $x$ and a matrix $A$ satisfying $\delta_{K+1}=t$ for which OMP may fail to recover the signal $\boldsymbol{x}$ in $K$ iterations. Moreover, the constraint on the minimum magnitude of the nonzero elements of $x$ is weaker than existing results.

Index Terms-Compressed sensing (CS), restricted isometry property (RIP), orthogonal matching pursuit (OMP), support recovery.
\end{abstract}

\section{INTRODUCTION}

In compressed sensing (CS), we usually observe the following linear model [1]-[4]:

$$
\boldsymbol{y}=\boldsymbol{A x}+\boldsymbol{v}
$$

where $\boldsymbol{x} \in \mathbb{R}^{n}$ is an unknown $K$-sparse signal, (i.e., $|\operatorname{supp}(\boldsymbol{x})| \leq K$, where $\operatorname{supp}(\boldsymbol{x})=\left\{i: x_{i} \neq 0\right\}$ is the support of $\boldsymbol{x}$ and $|\operatorname{supp}(\boldsymbol{x})|$ is the cardinality of $\operatorname{supp}(\boldsymbol{x})), \boldsymbol{A} \in \mathbb{R}^{m \times n}$ (with $m \ll n$ ) is a known sensing matrix, $\boldsymbol{v} \in \mathbb{R}^{m}$ is a noise vector, and $\boldsymbol{y} \in \mathbb{R}^{m}$ is the observation vector. There are many types of noises, for example, the $l_{2}$ bounded noise $\left(\|\boldsymbol{v}\|_{2} \leq \epsilon\right.$ for some constant $\left.\epsilon\right)$ [5]-[7], the $l_{\infty}$ bounded noise ( $\left.\left\|\boldsymbol{A}^{T} \boldsymbol{v}\right\|_{\infty} \leq \epsilon\right)$ [8], and Gaussian noise $\left(v_{i} \sim \mathcal{N}\left(0, \sigma^{2}\right)\right)$ [9]. In this paper, we consider only the $l_{2}$ bounded noise.

One of the central goals of CS is to recover the signal $\boldsymbol{x}$ based on the sensing matrix $\boldsymbol{A}$ and measurement $\boldsymbol{y}$. It

J. Wen is with College of Information Engineering and Shenzhen Key Lab of Media Security, Shenzhen University, Shenzhen 518060, China. He is also with ENS de Lyon, LIP, Lyon 69007, France, (e-mail: jwen@math.mcgill.ca). Z. Zhou is with School of Mathematics, Southwest Jiaotong University, Chengdu 610031, China, (e-mail: zzc@home.swjtu.edu.cn).

J. Wang is with Dept. of Electrical \& Computer Engineering, Seoul National University, Seoul 151742, Korea, (e-mail: wangjianeee@gmail.com).

$\mathrm{X}$. Tang is with Information Security and National Computing Gric Laboratory, Southwest Jiaotong University, Chengdu 610031, China, (e-mail: xhutang@swjtu.edu.cn).

Q. Mo is with Dept. of Mathematics, Zhejiang University, Hangzhou 310027, China, (e-mail: moqun@zju.edu.cn).

This work was supported by "Programme Avenir Lyon Saint-Etienne de l'Université de Lyon" in the framework of the programme "Inverstissements d'Avenir" (ANR-11-IDEX-0007), ANR through the HPAC project under Grant ANR 11 BS02 013, the NSF of China under grant 11271010, 11531013 and 61472024, and the fundamental research funds for Central Universities. has been revealed that under appropriate constraints on $\boldsymbol{A}$, reliable recovery of $\boldsymbol{x}$ can be achieved via properly designed algorithms (see, e.g., [10], [11]). Orthogonal matching pursuit (OMP) [12] is a widely used algorithm for recovering sparse signals. For any set $S \subset\{1,2, \ldots, n\}$, let $\boldsymbol{A}_{S}$ be the submatrix of $\boldsymbol{A}$ that contains only the columns indexed by $S$, and $\boldsymbol{x}_{S}$ be the subvector of $\boldsymbol{x}$ that contains only the entries indexed by $S$. The OMP algorithm is described in Algorithm 1 [12].

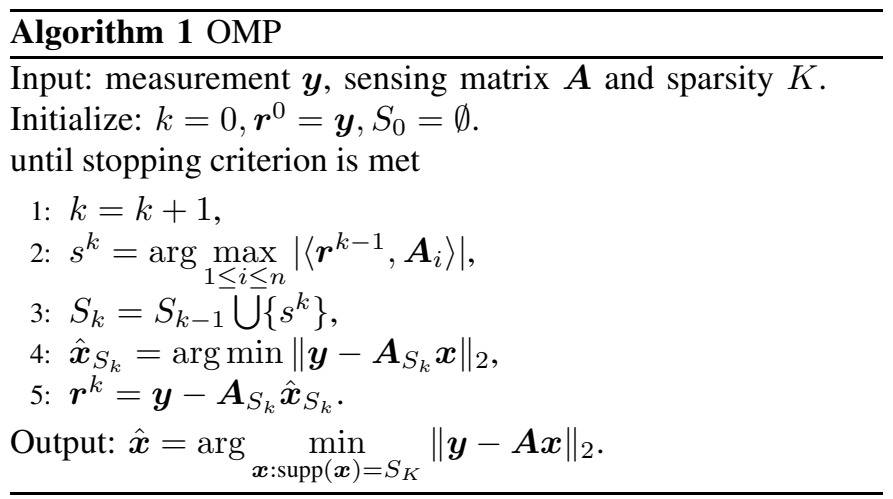

A commonly used framework for analyzing CS recovery algorithms is the restricted isometry property (RIP) [1]. For any $m \times n$ matrix $\boldsymbol{A}$ and any integer $K, 1 \leq K \leq n$, the order $K$ restricted isometry constant (RIC) $\delta_{K}$ is defined as the smallest constant such that

$$
\left(1-\delta_{K}\right)\|\boldsymbol{x}\|_{2}^{2} \leq\|\boldsymbol{A} \boldsymbol{x}\|_{2}^{2} \leq\left(1+\delta_{K}\right)\|\boldsymbol{x}\|_{2}^{2}
$$

for all $K$-sparse vectors $\boldsymbol{x}$.

Many RIC-based conditions have been proposed to ensure exact recovery of sparse signals with OMP in the noise-free case. It has respectively been shown in [13] and [14] that $\delta_{K+1}<\frac{1}{3 \sqrt{K}}$ and $\delta_{K+1}<\frac{1}{(1+\sqrt{2}) \sqrt{K}}$ are sufficient for OMP to recover any $K$-sparse $\boldsymbol{x}$ in $K$ iterations. The condition has been improved to $\delta_{K+1}<\frac{1}{1+\sqrt{K}}$ in [15], [16], and further improved to $\delta_{K+1}<\frac{\sqrt{4 K+1}-1}{2 K}$ in [17]. Recently, it is shown in [18] that if $\delta_{K+1}<\frac{1}{\sqrt{K+1}}$, then OMP exactly recovers the $K$-sparse signal $\boldsymbol{x}$ in $K$ iterations. On the other hand, it was conjectured in [19] that there exist a matrix $\boldsymbol{A}$ with $\delta_{K+1} \leq \frac{1}{\sqrt{K}}$ and a $K$-sparse $\boldsymbol{x}$ such that OMP fails to recover $\boldsymbol{x}$ in $K$ iterations. Examples provided in [15], [16] confirmed this conjecture. Later, the example in [20] showed that for any given positive integer $K \geq 2$ and for any given $t$ satisfying $\frac{1}{\sqrt{K+1}} \leq t<1$, there always exist a $K$-sparse $\boldsymbol{x}$ and a matrix $A$ satisfies the RIP of order $K+1$ with $\delta_{K+1}=t$ such that 
OMP may fail to recover the signal $x$ in $K$ iterations. In other words, the sufficient condition for recovering $\boldsymbol{x}$ cannot be weaker than $\delta_{K+1}<\frac{1}{\sqrt{K+1}}$. Thus, $\delta_{K+1}<\frac{1}{\sqrt{K+1}}$ [18] is a sharp condition guaranteeing exact recovery of $K$-sparse signals with the OMP algorithm.

For the noisy case, we are interested in recovering the support of $\boldsymbol{x}$, since the signal can be estimated by an ordinary least squares regression on the recovered support [8]. It was shown in [21] that under some condition on the minimum magnitude of the nonzero elements of $\boldsymbol{x}, \delta_{K+1}<\frac{1}{\sqrt{K}+3}$ is sufficient for exact recovery of $\operatorname{supp}(\boldsymbol{x})$ with OMP under the $l_{2}$ bounded noise. This condition has been improved to $\delta_{K+1}<\frac{1}{\sqrt{K}+1}$ [22]. And the best existing condition in terms of $\delta_{K+1}$ is $\delta_{K+1}<\frac{\sqrt{4 K+1}-1}{2 K}$ [17].

In this paper, we investigate the RIP condition and the minimum magnitude of the nonzero elements of the $K$-sparse signal $\boldsymbol{x}$ that guarantee the recovery of $\operatorname{supp}(\boldsymbol{x})$ with OMP under the $l_{2}$ bounded noise $\left(\left\|\boldsymbol{r}^{k}\right\| \leq \epsilon\right)$. We show that if $\boldsymbol{A}$ and $\boldsymbol{v}$ in (1) respectively satisfy the RIP of order $K+1$ with

$$
\delta_{K+1}<\frac{1}{\sqrt{K+1}},
$$

then the OMP algorithm with stopping criterion $\left\|\boldsymbol{r}^{k}\right\| \leq \epsilon$ exactly recovers $\operatorname{supp}(\boldsymbol{x})$ provided that

$$
\min _{i \in \operatorname{supp}(\boldsymbol{x})}\left|x_{i}\right|>\frac{2 \epsilon}{1-\sqrt{K+1} \delta_{K+1}} .
$$

By the aforementioned analysis, condition (3) is sharp in terms of $\delta_{K+1}$. We also show that condition (4) on $\min _{i \in \operatorname{supp}(\boldsymbol{x})}\left|x_{i}\right|$ is also weaker than existing results.

The rest of the paper is organized as follows. In section II, we present a sharp condition for the exact support recovery of the $K$-sparse signal $\boldsymbol{x}$ by OMP under the $l_{2}$ bounded noise. In section III, we compare our sufficient condition with existing ones. Finally, we summarize this paper in section IV.

Notation: Let $\mathbb{R}$ be the real field. Boldface lowercase letters denote column vectors, and boldface uppercase letters denote matrices, e.g., $\boldsymbol{x} \in \mathbb{R}^{n}$ and $\boldsymbol{A} \in \mathbb{R}^{m \times n}$. Let $\Omega=\operatorname{supp}(\boldsymbol{x})$, then $|\Omega| \leq K$ for any $K$-sparse signal $\boldsymbol{x}$, where $|\Omega|$ is the cardinality of $\Omega$. Let $\Omega \backslash S=\{k \mid k \in \Omega, k \notin S\}$ for set $S$. Let $\Omega^{c}$ and $S^{c}$ be the complement of $\Omega$ and $S$, i.e., $\Omega^{c}=$ $\{1,2, \ldots, n\} \backslash \Omega$, and $S^{c}=\{1,2, \ldots, n\} \backslash S$. Let $\boldsymbol{A}_{S}$ be the submatrix of $\boldsymbol{A}$ that contains only the columns indexed by $\boldsymbol{S}$, and $\boldsymbol{x}_{S}$ be the subvector of $\boldsymbol{x}$ that contains only the entries indexed by $\boldsymbol{S}$, and $\boldsymbol{A}_{S}^{T}$ be the transpose of $\boldsymbol{A}_{S}$. For full column rank matrix $\boldsymbol{A}_{S}$, let $\boldsymbol{P}_{S}=\boldsymbol{A}_{S}\left(\boldsymbol{A}_{S}^{T} \boldsymbol{A}_{S}\right)^{-1} \boldsymbol{A}_{S}^{T}$ and $\boldsymbol{P}_{S}^{\perp}=\boldsymbol{I}-$ $\boldsymbol{P}_{S}$ denote the projector and orthogonal complement projector on the column space of $\boldsymbol{A}_{S}$, respectively.

\section{A SHARP CONDITION FOR EXACT SUPPORT RECOVERY UNDER THE $l_{2}$ BOUNDED NOISE}

In this section, we show that if $\boldsymbol{A}$ satisfies the RIP of order $K+1$ with $\delta_{K+1}<\frac{1}{\sqrt{K+1}}$, then under some condition on the minimum magnitude of the nonzero elements of the $K$-sparse signal $\boldsymbol{x}, \operatorname{supp}(\boldsymbol{x})$ can be exactly recovered by OMP under the $l_{2}$ bounded noise.
Before introducing our main result, we present the following lemma which is inspired by [18].

Lemma 1: Suppose that $\boldsymbol{A}$ in (1) satisfies the RIP of order $K+1$ with $0 \leq \delta_{K+1}<1$. Let $S$ be a subset of $\Omega=\operatorname{supp}(\boldsymbol{x})$ with $|S|<|\Omega|$. Then,

$$
\begin{aligned}
& \left\|\boldsymbol{A}_{\Omega \backslash S}^{T} \boldsymbol{P}_{S}^{\perp} \boldsymbol{A}_{\Omega \backslash S} \boldsymbol{x}_{\Omega \backslash S}\right\|_{\infty}-\left\|\boldsymbol{A}_{\Omega^{c}}^{T} \boldsymbol{P}_{S}^{\perp} \boldsymbol{A}_{\Omega \backslash S} \boldsymbol{x}_{\Omega \backslash S}\right\|_{\infty} \\
\geq & \frac{\left(1-\sqrt{|\Omega|-|S|+1} \delta_{|\Omega|+1}\right)\left\|\boldsymbol{x}_{\Omega \backslash S}\right\|_{2}}{\sqrt{|\Omega|-|S|}} .
\end{aligned}
$$

Due to the page limit, we skip the proof of Lemma 1 and only give an easily-checked example to explain the lemma. Interested readers are referred to [23] for a detailed proof.

Example: Let $K=2$ and $S=\{1\}$. For $0 \leq \delta<1$, let

$$
\boldsymbol{A}=\left[\begin{array}{ccc}
\sqrt{1+\delta} & 0 & 0 \\
0 & \sqrt{1-\delta} & 0 \\
0 & 0 & \sqrt{1+\delta}
\end{array}\right] \text { and } \boldsymbol{x}=\left[\begin{array}{l}
1 \\
1 \\
0
\end{array}\right]
$$

then $\boldsymbol{x}$ is 2 -sparse and $\Omega=\{1,2\}$. It is clear that

$$
\boldsymbol{P}_{S}^{\perp}=\boldsymbol{P}_{\{1\}}^{\perp}=\left[\begin{array}{ccc}
0 & 0 & 0 \\
0 & 1 & 0 \\
0 & 0 & 1
\end{array}\right] .
$$

Also, it is easily checked that $\delta_{3}=\delta$ and

$$
\begin{aligned}
& \left\|\boldsymbol{A}_{\Omega \backslash S}^{T} \boldsymbol{P}_{S}^{\perp} \boldsymbol{A}_{\Omega \backslash S} \boldsymbol{x}_{\Omega \backslash S}\right\|_{\infty}-\left\|\boldsymbol{A}_{\Omega^{c}}^{T} \boldsymbol{P}_{S}^{\perp} \boldsymbol{A}_{\Omega \backslash S} \boldsymbol{x}_{\Omega \backslash S}\right\|_{\infty} \\
= & \left|\boldsymbol{A}_{\{2\}}^{T} \boldsymbol{P}_{\{1\}}^{\perp} \boldsymbol{A}_{\{2\}} \boldsymbol{x}_{\{2\}}\right|-\left|\boldsymbol{A}_{\{3\}}^{T} \boldsymbol{P}_{\{1\}}^{\perp} \boldsymbol{A}_{\{2\}} \boldsymbol{x}_{\{2\}}\right| \\
= & 1-\delta .
\end{aligned}
$$

One can show that

$$
\frac{\left(1-\sqrt{|\Omega|-|S|+1} \delta_{|\Omega|+1}\right)\left\|\boldsymbol{x}_{\Omega \backslash S}\right\|_{2}}{\sqrt{|\Omega|-|S|}}=1-\sqrt{2} \delta .
$$

By the aforementioned two equations, (5) obviously holds in this case.

Since $|\Omega| \leq K$, from (5) it is not hard to see that under (3), the right-hand side of (5) is positive.

The following theorem gives a sufficient condition for exactly recovering $\operatorname{supp}(\boldsymbol{x})$ with OMP.

Theorem 1: Suppose that $\boldsymbol{A}$ and $\boldsymbol{v}$ in (1) satisfy (3) and $\|\boldsymbol{v}\|_{2} \leq \epsilon$, respectively. Then the OMP algorithm with stopping criterion $\left\|\boldsymbol{r}^{k}\right\| \leq \epsilon$ exactly recovers the support $\Omega$ of the $K$ sparse signal $\boldsymbol{x}$ provided that

$$
\min _{i \in \Omega}\left|x_{i}\right|>\frac{2 \epsilon}{1-\sqrt{K+1} \delta_{K+1}} .
$$

Before proving Theorem 1, we introduce three lemmas that are useful for our analysis.

Lemma 2 ( [1]): If $\boldsymbol{A}$ satisfies the RIP of orders $k_{1}$ and $k_{2}$ with $k_{1}<k_{2}$, then $\delta_{k_{1}} \leq \delta_{k_{2}}$.

Lemma 3 ( [24]): Let $\boldsymbol{A}$ satisfy the RIP of order $k$ and $S$ be a set with $|S| \leq k$, then for any $\boldsymbol{x} \in \mathbb{R}^{m}$,

$$
\left\|\boldsymbol{A}_{S}^{T} \boldsymbol{x}\right\|_{2}^{2} \leq\left(1+\delta_{k}\right)\|\boldsymbol{x}\|_{2}^{2}
$$


Lemma 4 ( [25]): Let sets $S_{1}, S_{2}$ satisfy $\left|S_{2} \backslash S_{1}\right| \geq 1$ and matrix $\boldsymbol{A}$ satisfy the RIP of order $\left|S_{1} \cup S_{2}\right|$, then for any vector $\boldsymbol{x} \in \mathbb{R}^{\left|S_{2} \backslash S_{1}\right|}$,

$\left(1-\delta_{\left|S_{1} \cup S_{2}\right|}\right)\|\boldsymbol{x}\|_{2}^{2} \leq\left\|\boldsymbol{P}_{S_{1}}^{\perp} \boldsymbol{A}_{S_{2} \backslash S_{1}} \boldsymbol{x}\right\|_{2}^{2} \leq\left(1+\delta_{\left|S_{1} \cup S_{2}\right|}\right)\|\boldsymbol{x}\|_{2}^{2}$.

Proof of Theorem 1. We prove the theorem in two steps. First, we show that OMP selects correct indexes in all iterations. In the second step, we prove that the algorithm performs exactly $|\Omega|$ iterations before stopping.

We prove the first step by induction. Suppose that OMP selects correct indexes in the first $k-1$ iterations, i.e., $S_{k-1} \subseteq \Omega$. Then, we will show that the OMP algorithm also selects a correct index in the $k$-th iteration, that is, $s^{k} \in \Omega$. Here, we assume $1 \leq k \leq|\Omega|$, thus the proof for the first selection is contained in the case that $k=1$. Also, the induction assumption $S_{k-1} \subseteq \Omega$ holds in this case since $S_{0}=\emptyset$.

Obviously, for $i \in S_{k-1},\left\langle\boldsymbol{r}^{k-1}, \boldsymbol{A}_{i}\right\rangle=0$. Thus by line 2 of Algorithm 1, to show $s^{k} \in \Omega$, it suffices to show

$$
\max _{i \in \Omega \backslash S_{k-1}}\left|\left\langle\boldsymbol{r}^{k-1}, \boldsymbol{A}_{i}\right\rangle\right|>\max _{j \in \Omega^{c}}\left|\left\langle\boldsymbol{r}^{k-1}, \boldsymbol{A}_{j}\right\rangle\right| .
$$

From line 4 of Algorithm 1, we have

$$
\hat{\boldsymbol{x}}_{S_{k-1}}=\left(\boldsymbol{A}_{S_{k-1}}^{T} \boldsymbol{A}_{S_{k-1}}\right)^{-1} \boldsymbol{A}_{S_{k-1}}^{T} \boldsymbol{y} .
$$

Thus, by line 5 of Algorithm 1 and (8), we have

$$
\begin{aligned}
\boldsymbol{r}^{k-1} & =\boldsymbol{y}-\boldsymbol{A}_{S_{k-1}} \hat{\boldsymbol{x}}_{S_{k-1}} \\
& =\left(\boldsymbol{I}-\boldsymbol{A}_{S_{k-1}}\left(\boldsymbol{A}_{S_{k-1}}^{T} \boldsymbol{A}_{S_{k-1}}\right)^{-1} \boldsymbol{A}_{S_{k-1}}^{T}\right) \boldsymbol{y} \\
& \stackrel{(a)}{=} \boldsymbol{P}_{S_{k-1}}^{\perp}(\boldsymbol{A} \boldsymbol{x}+\boldsymbol{v}) \\
& \stackrel{(b)}{=} \boldsymbol{P}_{S_{k-1}}^{\perp}\left(\boldsymbol{A}_{\Omega} \boldsymbol{x}_{\Omega}+\boldsymbol{v}\right) \\
& \stackrel{(c)}{=} \boldsymbol{P}_{S_{k-1}}^{\perp}\left(\boldsymbol{A}_{S_{k-1}} \boldsymbol{x}_{S_{k-1}}+\boldsymbol{A}_{\Omega \backslash S_{k-1}} \boldsymbol{x}_{\Omega \backslash S_{k-1}}+\boldsymbol{v}\right) \\
& \stackrel{(d)}{=} \boldsymbol{P}_{S_{k-1}}^{\perp} \boldsymbol{A}_{\Omega \backslash S_{k-1}} \boldsymbol{x}_{\Omega \backslash S_{k-1}}+\boldsymbol{P}_{S_{k-1}}^{\perp} \boldsymbol{v},
\end{aligned}
$$

where (a), (b), (c) and (d) follow from the definition of $\boldsymbol{P}_{S_{k-1}}^{\perp}$, the fact that $\Omega=\operatorname{supp}(\boldsymbol{x})$, the induction assumption $S_{k-1} \subseteq$ $\Omega$, and $\boldsymbol{P}_{S_{k-1}}^{\perp} \boldsymbol{A}_{S_{k-1}}=\mathbf{0}$, respectively.

Then it follows from (9) that

$$
\begin{aligned}
& \max _{i \in \Omega \backslash S_{k-1}}\left|\left\langle\boldsymbol{r}^{k-1}, \boldsymbol{A}_{i}\right\rangle\right| \\
= & \left\|\boldsymbol{A}_{\Omega \backslash S_{k-1}}^{T}\left(\boldsymbol{P}_{S_{k-1}}^{\perp} \boldsymbol{A}_{\Omega \backslash S_{k-1}} \boldsymbol{x}_{\Omega \backslash S_{k-1}}+\boldsymbol{P}_{S_{k-1}}^{\perp} \boldsymbol{v}\right)\right\|_{\infty} \\
\geq & \left\|\boldsymbol{A}_{\Omega \backslash S_{k-1}}^{T} \boldsymbol{P}_{S_{k-1}}^{\perp} \boldsymbol{A}_{\Omega \backslash S_{k-1}} \boldsymbol{x}_{\Omega \backslash S_{k-1}}\right\|_{\infty} \\
& -\left\|\boldsymbol{A}_{\Omega \backslash S_{k-1}}^{T} \boldsymbol{P}_{S_{k-1}}^{\perp} \boldsymbol{v}\right\|_{\infty},
\end{aligned}
$$

and

$$
\begin{aligned}
& \max _{j \in \Omega^{c}}\left|\left\langle\boldsymbol{r}^{k-1}, \boldsymbol{A}_{j}\right\rangle\right| \\
= & \left\|\boldsymbol{A}_{\Omega^{c}}^{T}\left(\boldsymbol{P}_{S_{k-1}}^{\perp} \boldsymbol{A}_{\Omega \backslash S_{k-1}} \boldsymbol{x}_{\Omega \backslash S_{k-1}}+\boldsymbol{P}_{S_{k-1}}^{\perp} \boldsymbol{v}\right)\right\|_{\infty} \\
\leq & \left\|\boldsymbol{A}_{\Omega^{c}}^{T} \boldsymbol{P}_{S_{k-1}}^{\perp} \boldsymbol{A}_{\Omega \backslash S_{k-1}} \boldsymbol{x}_{\Omega \backslash S_{k-1}}\right\|_{\infty}+\left\|\boldsymbol{A}_{\Omega^{c}}^{T} \boldsymbol{P}_{S_{k-1}}^{\perp} \boldsymbol{v}\right\|_{\infty} .
\end{aligned}
$$

Therefore, from (10) and (11), to show (7), it suffices to show

$$
\begin{aligned}
& \left\|\boldsymbol{A}_{\Omega \backslash S_{k-1}}^{T} \boldsymbol{P}_{S_{k-1}}^{\perp} \boldsymbol{A}_{\Omega \backslash S_{k-1}} \boldsymbol{x}_{\Omega \backslash S_{k-1}}\right\|_{\infty} \\
- & \left\|\boldsymbol{A}_{\Omega^{c}}^{T} \boldsymbol{P}_{S_{k-1}}^{\perp} \boldsymbol{A}_{\Omega \backslash S_{k-1}} \boldsymbol{x}_{\Omega \backslash S_{k-1}}\right\|_{\infty} \\
> & \left\|\boldsymbol{A}_{\Omega \backslash S_{k-1}}^{T} \boldsymbol{P}_{S_{k-1}}^{\perp} \boldsymbol{v}\right\|_{\infty}+\left\|\boldsymbol{A}_{\Omega^{c}}^{T} \boldsymbol{P}_{S_{k-1}}^{\perp} \boldsymbol{v}\right\|_{\infty} .
\end{aligned}
$$

By induction assumption $S_{k-1} \subseteq \Omega$, we have

$$
\left|\operatorname{supp}\left(\boldsymbol{x}_{\Omega \backslash S_{k-1}}\right)\right|=|\Omega|+1-k .
$$

Thus,

$$
\begin{aligned}
\left\|\boldsymbol{x}_{\Omega \backslash S_{k-1}}\right\|_{2} & \geq \sqrt{|\Omega|+1-k} \min _{i \in \Omega \backslash S_{k-1}}\left|x_{i}\right| \\
& \geq \sqrt{|\Omega|+1-k} \min _{i \in \Omega}\left|x_{i}\right| .
\end{aligned}
$$

In the following, we give a lower bound on the left-hand side of (12). Since $S_{k-1} \subseteq \Omega$ and $\left|S_{k-1}\right|=k-1$, using Lemma 1 , we have

$$
\begin{aligned}
&\left\|\boldsymbol{A}_{\Omega \backslash S_{k-1}}^{T} \boldsymbol{P}_{S_{k-1}}^{\perp} \boldsymbol{A}_{\Omega \backslash S_{k-1}} \boldsymbol{x}_{\Omega \backslash S_{k-1}}\right\|_{\infty} \\
&-\left\|\boldsymbol{A}_{\Omega^{c}}^{T} \boldsymbol{P}_{S_{k-1}}^{\perp} \boldsymbol{A}_{\Omega \backslash S_{k-1}} \boldsymbol{x}_{\Omega \backslash S_{k-1}}\right\|_{\infty} \\
& \geq \frac{\left(1-\sqrt{|\Omega|-k+2} \delta_{|\Omega|+1}\right)\left\|\boldsymbol{x}_{\Omega \backslash S_{k-1}}\right\|_{2}}{\sqrt{|\Omega|+1-k}} \\
& \stackrel{(a)}{\geq} \frac{\left(1-\sqrt{K+1} \delta_{|\Omega|+1}\right)\left\|\boldsymbol{x}_{\Omega \backslash S_{k-1}}\right\|_{2}}{\sqrt{|\Omega|+1-k}} \\
& \stackrel{(b)}{\geq} \frac{\left(1-\sqrt{K+1} \delta_{K+1}\right)\left\|\boldsymbol{x}_{\Omega \backslash S_{k-1}}\right\|_{2}}{\sqrt{|\Omega|+1-k}} \\
& \stackrel{(c)}{\geq}\left(1-\sqrt{K+1} \delta_{K+1}\right) \min _{i \in \Omega}\left|x_{i}\right|,
\end{aligned}
$$

where (a) is because $k \geq 1$ and $\boldsymbol{x}$ is $K$-sparse (i.e., $|\Omega| \leq K$ ); (b) follows from Lemma 2; and (c) follows from (3) and (14).

Next, we give an upper bound for the right-hand side of (12). Clearly there exist $i_{0} \in \Omega \backslash S_{k-1}$ and $j_{0} \in \Omega^{c}$ such that

$$
\begin{aligned}
\left\|\boldsymbol{A}_{\Omega \backslash S_{k-1}}^{T} \boldsymbol{P}_{S_{k-1}}^{\perp} \boldsymbol{v}\right\|_{\infty} & =\left|\boldsymbol{A}_{i_{0}}^{T} \boldsymbol{P}_{S_{k-1}}^{\perp} \boldsymbol{v}\right|, \\
\left\|\boldsymbol{A}_{\Omega^{c}}^{T} \boldsymbol{P}_{S_{k-1}}^{\perp} \boldsymbol{v}\right\|_{\infty} & =\left|\boldsymbol{A}_{j_{0}}^{T} \boldsymbol{P}_{S_{k-1}}^{\perp} \boldsymbol{v}\right| .
\end{aligned}
$$

Therefore

$$
\begin{aligned}
& \left\|\boldsymbol{A}_{\Omega \backslash S_{k-1}}^{T} \boldsymbol{P}_{S_{k-1}}^{\perp} \boldsymbol{v}\right\|_{\infty}+\left\|\boldsymbol{A}_{\Omega^{c}}^{T} \boldsymbol{P}_{S_{k-1}}^{\perp} \boldsymbol{v}\right\|_{\infty} \\
& =\left|\boldsymbol{A}_{i_{0}}^{T} \boldsymbol{P}_{S_{k-1}}^{\perp} \boldsymbol{v}\right|+\left|\boldsymbol{A}_{j_{0}}^{T} \boldsymbol{P}_{S_{k-1}}^{\perp} \boldsymbol{v}\right| \\
& =\left\|\boldsymbol{A}_{i_{0} \cup j_{0}}^{T} \boldsymbol{P}_{S_{k-1}}^{\perp} \boldsymbol{v}\right\|_{1} \\
& \stackrel{(a)}{\leq} \sqrt{2}\left\|\boldsymbol{A}_{i_{0} \cup j_{0}}^{T} \boldsymbol{P}_{S_{k-1}}^{\perp} \boldsymbol{v}\right\|_{2} \\
& \stackrel{(b)}{\leq} \sqrt{2\left(1+\delta_{K+1}\right)}\left\|\boldsymbol{P}_{S_{k-1}}^{\perp} \boldsymbol{v}\right\|_{2} \\
& \leq \sqrt{2\left(1+\delta_{K+1}\right)} \epsilon,
\end{aligned}
$$

where (a) is because $\boldsymbol{A}_{i_{0} \cup j_{0}}^{T} \boldsymbol{P}_{S_{k-1}}^{\perp} \boldsymbol{v}$ is a $2 \times 1$ vector, (b) follows from Lemma 3 , and (c) is because

$$
\left\|\boldsymbol{P}_{S_{k-1}}^{\perp} \boldsymbol{v}\right\|_{2} \leq\left\|\boldsymbol{P}_{S_{k-1}}^{\perp}\right\|\left\|_{2}\right\| \boldsymbol{v}\left\|_{2} \leq\right\| \boldsymbol{v} \|_{2} \leq \epsilon .
$$


Finally, from (15) and (18), (12) (or equivalently (7)) is guaranteed by

$$
\left(1-\sqrt{K+1} \delta_{K+1}\right) \min _{i \in \Omega}\left|x_{i}\right|>\sqrt{2\left(1+\delta_{K+1}\right)} \epsilon .
$$

from which we obtain (3). Therefore, under (6), the OMP algorithm selects a correct index in each iteration.

Now we proceed to the second step of our proof. We show that the OMP algorithm performs exactly $|\Omega|$ iterations before stopping. That is, $\left\|\boldsymbol{r}^{k}\right\|_{2}>\epsilon$ for $1 \leq k<|\Omega|$ and $\left\|\boldsymbol{r}^{|\Omega|}\right\|_{2} \leq \epsilon$.

Since the OMP algorithm selects a correct index in each iteration under (6), by (9), we have that for $1 \leq k<|\Omega|$,

$$
\begin{aligned}
\left\|\boldsymbol{r}^{k}\right\|_{2} & =\left\|\boldsymbol{P}_{S_{k}}^{\perp} \boldsymbol{A}_{\Omega \backslash S_{k}} \boldsymbol{x}_{\Omega \backslash S_{k}}+\boldsymbol{P}_{S_{k}}^{\perp} \boldsymbol{v}\right\|_{2} \\
& \geq\left\|\boldsymbol{P}_{S_{k}}^{\perp} \boldsymbol{A}_{\Omega \backslash S_{k}} \boldsymbol{x}_{\Omega \backslash S_{k}}\right\|_{2}-\left\|\boldsymbol{P}_{S_{k}}^{\perp} \boldsymbol{v}\right\|_{2} \\
& \stackrel{(a)}{\geq}\left\|\boldsymbol{P}_{S_{k}}^{\perp} \boldsymbol{A}_{\Omega \backslash S_{k}} \boldsymbol{x}_{\Omega \backslash S_{k}}\right\|_{2}-\epsilon \\
& \stackrel{(b)}{\geq} \sqrt{1-\delta_{|\Omega|}}\left\|\boldsymbol{x}_{\Omega \backslash \boldsymbol{S}_{k}}\right\|_{2}-\epsilon \\
& \stackrel{(c)}{\geq} \sqrt{1-\delta_{K+1}} \sqrt{|\Omega|-k} \min _{i \in \Omega}\left|x_{i}\right|-\epsilon \\
& \geq \sqrt{1-\delta_{K+1}} \min _{i \in \Omega}\left|x_{i}\right|-\epsilon,
\end{aligned}
$$

where (a) is from (19); (b) is from Lemma 4; and (c) follows from Lemma 2 and (14). Thus, if

$$
\min _{i \in \Omega}\left|x_{i}\right|>\frac{2 \epsilon}{\sqrt{1-\delta_{K+1}}},
$$

then $\left\|\boldsymbol{r}^{k}\right\|_{2}>\epsilon$ for each $1 \leq k<\Omega$.

Furthermore, by noting that

$$
1-\sqrt{K+1} \delta_{K+1} \leq 1-\delta_{K+1} \leq \sqrt{1-\delta_{K+1}} .
$$

we have

$$
\frac{2 \epsilon}{1-\sqrt{K+1} \delta_{K+1}} \geq \frac{2 \epsilon}{\sqrt{1-\delta_{K+1}}} .
$$

This, together with (21), implies that if (6) holds, $\left\|\boldsymbol{r}^{k}\right\|_{2}>\epsilon$ for each $1 \leq k<\Omega$. In other words, the OMP algorithm does not terminate before the $|\Omega|$-th iteration.

Similarly, by (9),

$$
\begin{aligned}
\left\|\boldsymbol{r}^{|\Omega|}\right\|_{2} & =\left\|\boldsymbol{P}_{S_{|\Omega|}}^{\perp} \boldsymbol{A}_{\Omega \backslash S_{|\Omega|}} \boldsymbol{x}_{\Omega \backslash S_{|\Omega|}}+\boldsymbol{P}_{S_{|\Omega|}^{\perp}}^{\perp} \boldsymbol{v}\right\|_{2} \\
& \stackrel{(a)}{=}\left\|\boldsymbol{P}_{S_{|\Omega|}}^{\perp} \boldsymbol{v}\right\|_{2} \stackrel{(b)}{\leq} \epsilon,
\end{aligned}
$$

where (a) is because $S_{|\Omega|}=|\Omega|$ and (b) follows from (19). Therefore, under stopping condition $\left\|\boldsymbol{r}^{k}\right\|_{2}>\epsilon$, the OMP algorithm performs $|\Omega|$ iterations before stopping. This completes the proof.

From Theorem 1, if $\epsilon=0$, then $\|\boldsymbol{v}\|_{2}=0$ and (6) holds. Hence, $\operatorname{supp}(\boldsymbol{x})$ can be exactly recovered in $|\operatorname{supp}(\boldsymbol{x})|$ iterations if $\delta_{K+1}$ satisfies (3). We thus have the following result, which is equivalent to [18, Theorem III.1].

Corollary 1: Suppose that $\boldsymbol{A}$ and $\boldsymbol{v}$ in (1) satisfy the RIP of order $K+1$ with $\delta_{K+1}$ satisfying (3) and $\|\boldsymbol{v}\|_{2}=0$, respectively. Then the OMP algorithm exactly recovers the $K$-sparse signal $\boldsymbol{x}$ in $K$ iterations.
The example in [20] showed that for any given positive integer $K \geq 2$ and for any $\frac{1}{\sqrt{K+1}} \leq t<1$, there always exist a $K$-sparse $\boldsymbol{x}$ and a matrix $\boldsymbol{A}$ satisfying the RIP of order $K+1$ with $\delta_{K+1}=t$ such that the OMP algorithm may fail to recover $\boldsymbol{x}$. Thus, the sufficient condition, given in Theorem 1 , is sharp in terms of $\delta_{K+1}$ for guaranteeing exact recovery of $\operatorname{supp}(\boldsymbol{x})$.

\section{COMPARISON WITH EXITING SUFFICIENT CONDITIONS}

In this section, we show that our sufficient condition given in Theorem 1 is weaker than existing sufficient conditions.

In [17], [22], $\boldsymbol{A}$ was assumed to be column normalized, i.e., $\left\|\boldsymbol{A}_{i}\right\|_{2}=1$ for $i=1,2, \ldots, n$. Note that Theorem 1 obviously holds if $\boldsymbol{A}$ is column normalized. In fact, our result in Theorem 1 outperforms those in [17], [21], [22] in terms of both $\delta_{K+1}$ and the requirement on $\min _{i \in \Omega}\left|x_{i}\right|$. For simplicity, we only compare our condition with the so far best result [17].

It was shown in [17] that if $\boldsymbol{A}$ in (1) is column normalized and satisfies the RIP of order $K+1$ with $\delta_{K+1}$ satisfying

$$
\delta_{K+1}<\frac{\sqrt{4 K+1}-1}{2 K}
$$

and $\boldsymbol{v}$ in (1) satisfies $\|\boldsymbol{v}\|_{2} \leq \epsilon$. Then the OMP algorithm with stopping criterion $\left\|\boldsymbol{r}^{k}\right\| \leq \epsilon$ exactly recovers the support $\Omega$ of the $K$-sparse signal $\boldsymbol{x}$ if

$$
\min _{i \in \Omega}\left|x_{i}\right|>\frac{\left(\sqrt{1+\delta_{K+1}}+1\right) \epsilon}{1-\delta_{K+1}-\sqrt{1-\delta_{K+1}} \sqrt{K} \delta_{K+1}} .
$$

By Theorem 1, to show our condition is better (weaker), we only need to show that

$$
\frac{\sqrt{4 K+1}-1}{2 K}<\frac{1}{\sqrt{K+1}}
$$

and that

$$
\frac{\left(\sqrt{1+\delta_{K+1}}+1\right) \epsilon}{1-\delta_{K+1}-\sqrt{1-\delta_{K+1}} \sqrt{K} \delta_{K+1}} \geq \frac{2 \epsilon}{1-\sqrt{K+1} \delta_{K+1}}
$$

for $\delta_{K+1}$ satisfying (3). In particular, if $\delta_{K+1} \neq 0$, then the strict inequality in (24) holds.

Clearly to show (23), it suffices to show

$$
\sqrt{(4 K+1)(K+1)}<2 K+\sqrt{K+1} .
$$

Equivalently,

$$
4 K^{2}+5 K+1<4 K^{2}+K+1+4 K \sqrt{K+1} .
$$

In fact, since $K \geq 1$, the above equation holds trivially, and hence (23) is true.

Next, we assume $\delta_{K+1} \neq 0$ satisfies (3) and then show the strict inequality in (24) holds. Since $\delta_{K+1} \neq 0$,

$$
\sqrt{1+\delta_{K+1}}+1>2 \text {. }
$$

Thus, it suffices to show

$$
1-\delta_{K+1}-\sqrt{1-\delta_{K+1}} \sqrt{K} \delta_{K+1}<1-\sqrt{K+1} \delta_{K+1},
$$


or equivalently,

$$
1+\sqrt{1-\delta_{K+1}} \sqrt{K}>\sqrt{K+1}
$$

Obviously, (25) holds if

$$
\sqrt{1-\delta_{K+1}}>\frac{\sqrt{K+1}-1}{\sqrt{K}}
$$

which is equivalent to

$$
\delta_{K+1}<\frac{2(\sqrt{K+1}-1)}{K} .
$$

Thus, a sufficient condition of (24) is

$$
\frac{1}{\sqrt{K+1}}<\frac{2(\sqrt{K+1}-1)}{K} \text {. }
$$

By some simple calculations, one can easily show that the aforementioned inequality holds. Therefore, the strict inequality in (24) holds if $\delta_{K+1} \neq 0$ satisfies (3).

\section{CONCLUSION}

In this paper, we have studied the condition for exact support recovery of sparse signals from noisy measurements with OMP. We have shown that if the sensing matrix $\boldsymbol{A}$ satisfies $\delta_{K+1}<\frac{1}{\sqrt{K+1}}$, then under some constraint on the minimum magnitude of the nonzero elements of the $K$-sparse signal $\boldsymbol{x}$, the support of the signal can be exactly recovered under the $l_{2}$ bounded noise. This condition is sharp in terms of $\delta_{K+1}$ and also the constraint on the minimum magnitude of the nonzero elements of $\boldsymbol{x}$ is weaker than existing ones.

\section{REFERENCES}

[1] E. J. Candés and T. Tao, "Decoding by linear programming," IEEE Trans. Inf. Theory, vol. 51, no. 12, pp. 4203-4215, 2005.

[2] D. L. Donoho, "Compressed sensing," IEEE Trans. Inf. Theory, vol. 52, no. 4, pp. 1289-1306, 2006.

[3] A. Cohen, W. Dahmen, and R. DeVore, "Compressed sensing and best $k$-term approximation,” J. Amer. Math. Soc., vol. 22, pp. 211-231, 2009.

[4] J. Wen, D. Li, and F. Zhu, "Stable recovery of sparse signals via $l_{p^{-}}$ minimization," Applied and Computational Harmonic Analysis, vol. 38, no. 1, pp. 161-176, 2015.

[5] J. J. Fuchs., "Recovery of exact sparse representations in the presence of bounded noise," IEEE Trans. Inf. Theory, vol. 51, no. 10, pp. 36013608, 2005.

[6] D. L. Donoho, M. Elad, and V. N. Temlyakov, "Stable recovery of sparse overcomplete representations in the presence of noise," IEEE Trans. Inf. Theory, vol. 52, pp. 6-18, 2006.

[7] E. J. Candés, "The restricted isometry property and its implications for compressed sensing," C. R. Acad. Sci. Paris, Ser. I, vol. 346, no. 11, pp. 589-592, 2008.

[8] T. Cai and L. Wang, "Orthogonal matching pursuit for sparse signal recovery with noise," IEEE Trans. Inf. Theory, vol. 57, pp. 4680-4688, 2011.

[9] E. J. Candés and T. Tao, "The dantzig selector: Statistical estimation when $p$ is much larger than n," Ann. Statist, vol. 35, pp. 2313-2351, 2007.

[10] E. J. Candés, J. Romberg, and T. Tao, "Stable signal recovery from incomplete and inaccurate measurements," Comm. Pure Appl. Math, vol. 59, pp. 1207-1223, 2006.

[11] Q. Mo and S. Li, "New bounds on the restricted isometry constant $\delta_{2 k}$," Appl. Comput. Harmon. Anal., vol. 31, pp. 460-468, 2011.

[12] J. A. Tropp and A. C. Gilbert, "Signal recovery from random measurements via orthogonal matching pursuit," IEEE Trans. Inf. Theory, vol. 53, no. 12, pp. 4655-4666, 2007.
[13] M. Davenport and M. Wakin, "Analysis of orthogonal matching pursuit using the restricted isometry property," IEEE Trans. Inf. Theory, vol. 56, no. 9, pp. 4395-4401, 2010.

[14] E. Liu and V. Temlyakov, "The orthogonal super greedy algorithm and applications in compressed sensing," IEEE Trans. Inf. Theory, vol. 58, no. 4, pp. 2040-2047, 2012.

[15] Q. Mo and S. Yi, "A remark on the restricted isometry property in orthogonal matching pursuit," IEEE Trans. Inf. Theory, vol. 58, no. 6, pp. 3654-3656, 2012.

[16] J. Wang and B. Shim, "On the recovery limit of sparse signals using orthogonal matching pursuit," IEEE Trans. Signal Process., vol. 60, no. 9, pp. 4973-4976, 2012.

[17] L.-H. Chang and J.-Y. Wu, "An improved RIP-based performance guarantee for sparse signal recovery via orthogonal matching pursuit," IEEE Trans. Inf. Theory, vol. 60, no. 9, pp. 707-710, 2014.

[18] Q. Mo, "A sharp restricted isometry constant bound of orthogonal matching pursuit," arXiv:1501.01708.

[19] M. Davenport and M. Wakin, "Subspace pursuit for compressive sensing signal reconstruction," IEEE Trans. Inf. Theory, vol. 55, no. 5, pp. 2230 2249, 2009.

[20] J. Wen, X. Zhu, and D. Li, "Improved bounds on the restricted isometry constant for orthogonal matching pursuit," Electronics Letters, vol. 49, pp. 1487-1489, 2013.

[21] Y. Shen and S. Li, "Sparse signals recovery from noisy measurements by orthogonal matching pursuit," Inverse Problems and Imaging, vol. 9, no. 1, pp. 231-238, 2015.

[22] R. Wu, W. Huang, and D. Chen, "The exact support recovery of sparse signals with noise via orthogonal matching pursuit," IEEE Signal Processing Letters, vol. 20, no. 4, pp. 403-406, 2013.

[23] J. Wen, Z. Zhou, J. Wang, X. Tang, and Q. Mo, "Sharp conditions for exact support recovery of sparse signals with noise via OMP," arXiv preprint arXiv: 1512.07248, 2015.

[24] D. Needel and J. A. Tropp, "CoSaMP: Iterative signal recovery from incomplete and inaccurate samples," Applied and Computational Harmonic Analysis, vol. 26, no. 3, pp. 301-321, 2009.

[25] Y. Shen, B. Li, W. Pan, and J. Li, "Analysis of generalized orthogonal matching pursuit using restricted isometry constant," Electron. Lett., vol. 50, no. 14, pp. 1020-1022, 2014. 